\title{
Relating function spaces to resourced function spaces
}

\author{
Lidia Sánchez-Gil \\ Universidad Complutense de \\ Madrid \\ lidiasg@mat.ucm.es
}

\author{
Mercedes \\ Hidalgo-Herrero \\ Universidad Complutense de \\ Madrid \\ mhidalgo@edu.ucm.es
}

\author{
Yolanda Ortega-Mallén \\ Universidad Complutense de \\ Madrid \\ yolanda@sip.ucm.es
}

\begin{abstract}
In order to prove the computational adequacy of the (operational) natural semantics for lazy evaluation with respect to a standard denotational semantics, Launchbury defines a resourced denotational semantics. This should be equivalent to the standard one when given infinite resources, but this fact cannot be so directly established, because each semantics produces values in a different domain. The values obtained by the standard semantics belong to the usual lifted function space $D=[D \rightarrow D]_{\perp}$, while those produced by the resourced semantics belong to $[C \rightarrow E]$ where $E$ satisfies the equation $E=[[C \rightarrow E] \rightarrow[C \rightarrow E]]_{\perp}$ and $C$ (the domain of resources) is a countable chain domain defined as the least solution of the domain equation $C=C_{\perp}$.

We propose a way to relate functional values in the standard lifted function space to functional values in the corresponding resourced function space. We first construct the initial solution for the domain equation $E=[[C \rightarrow E] \rightarrow$ $[C \rightarrow E]]_{\perp}$ following Abramsky's construction of the initial solution of $D=[D \rightarrow D]_{\perp}$. Then we define a "similarity" relation between values in the constructed domain and values in the standard lifted function space. This relation is inspired by Abramsky's applicative bisimulation.

Finally we prove the desired equivalence between the standard denotational semantics and the resourced semantics for the lazy $\lambda$-calculus.
\end{abstract}

\section{Categories and Subject Descriptors}

D.3.1 [Programming Languages]: Formal Definitions and Theory-semantics

\section{Keywords}

Domain theory, denotational semantics, $\lambda$-calculus.

\section{MOTIVATION}

There is a mismatch between the pure $\lambda$-calculus in the standard theory [4] and the practice of functional program-

Permission to make digital or hard copies of all or part of this work for personal or classroom use is granted without fee provided that copies are not made or distributed for profit or commercial advantage and that copies bear this notice and the full citation on the first page. To copy otherwise, to republish, to post on servers or to redistribute to lists, requires prior specific permission and/or a fee.

SAC'11 March 21-25, 2011, TaiChung, Taiwan.

Copyright 2011 ACM 978-1-4503-0113-8/11/03 ...\$10.00. ming, or more precisely, the lazy functional languages. This situation has been pointed out clearly by Abramsky in his seminal paper [1], where he proposes a "lazy" theory based on the notion of applicative transition systems and introduces a suitable domain equation, i.e., $D=[D \rightarrow D]_{\perp}$, which has a nontrivial initial solution that constitutes a canonical model for the family of lazy languages. The construction of this initial solution is detailed in [2]. We adapt this construction to the case where resources are added to the computational model, so that we obtain the initial solution for the domain equation $E=[[C \rightarrow E] \rightarrow[C \rightarrow E]]_{\perp}$, where $C$ is a countable chain domain defined as the least solution of the domain equation $C=C_{\perp}$.

"Resourced" domains like $E$ enable to define semantics that restrict the number of available resources. For instance, let us consider that only 60 seconds of CPU time are available and that each reduction needs one second; thus after 60 reductions the system gets blocked and the semantic value is $\perp$. Another example: Boudol, Curien and Lavatelli present in [5] a $\lambda$-calculus with resources to provide a control on the substitution process, so that a computation gets in deadlock when there are not enough resources to carry out all the substitutions. For this they use a different domain equation, namely $D=[\mathcal{M}(D) \rightarrow D]_{\perp}$, where $\mathcal{M}(D)$ stands for multisets of $D$.

The resourced domain $E$ was used by Launchbury in [7] to define a (denotational) resourced semantics that was introduced to prove the computational adequacy of his (operational) natural semantics for lazy evaluation. In that resourced semantics each syntactic level (in the term to be evaluated) requires the consumption of one resource. This mimics the derivation process for the natural semantics, where one operational rule is applied to eliminate each syntactic level. Hence, denotations that consume a finite number of resources correspond to finite derivations. Nevertheless, the construction of the initial solution for the domain equation $E=[[C \rightarrow E] \rightarrow[C \rightarrow E]]_{\perp}$ is not detailed in Launchbury's work, and we have found it described nowhere.

Our motivation for constructing an initial solution for $E=[[C \rightarrow E] \rightarrow[C \rightarrow E]]_{\perp}$ (let us call it CValue) is to define a correspondence between its elements and those of the lifted function space $D=[D \rightarrow D]_{\perp}$ (let us call it Value). This correspondence is necessary to prove that, provided an unbounded number of resources, the resourced semantics equals the standard denotational semantics. In [7] Launchbury simply states that both semantics compute the same values, but this is not exact because the values produced by the standard semantics belong to Value, while 
those obtained by the resourced semantics belong to CValue. How can be related functional values belonging to different semantic domains? The obvious answer is to observe their behaviour when applying them to any argument; but arguments and results are functional values too, thus some kind of recursive definition is needed. We are inspired by the applicative bisimulation defined by Abramsky in [1] as the limit of a sequence of relations, each one allowing to observe the applicative behaviour of functions until some fixed depth. But in Abramsky's applicative bisimulation the "experiments" are common to both sides of the simulation namely, they are syntactic terms - while in our case, they are again values belonging to different domains. Therefore, our version of the applicative bisimulation is in some way more general, and it can be useful for the study of simulations between distinct sets.

Summarizing, the contributions of this paper are: (1) the construction of the initial solution for the domain equation $E=[[C \rightarrow E] \rightarrow[C \rightarrow E]]_{\perp}$, where $C$ represents resources and satisfies the equation $C=C_{\perp}$, (2) the definition of a "similarity" relation between values in the constructed domain and values in the standard lifted function space $D=[D \rightarrow D]_{\perp}$, and (3) the proof of the equivalence between a standard denotational semantics and a resourced semantics for the lazy $\lambda$-calculus.

These results allow us to obtain a proof for the computational adequacy of Launchbury's natural semantics. As we have pointed out above, Launchbury just assumes that values obtained from both denotational semantics can be directly related, but the adequacy proof is not detailed. To the best of our knowledge, no complete and detailed proof of this adequacy has been published before. When trying to reproduce it we have discovered that it is not a straightforward proof, and that several subtle difficulties arise.

The adequacy result is of interest since Launchbury's natural semantics has been often cited and has inspired many other works as well as several extensions of his semantics, where the corresponding adequacy proofs have been obtained by adapting Launchbury's proof scheme, e.g., [14, 3, 15, 9, 11]. Moreover, the resourced function space and the similarity relation defined here can be useful in other contexts.

The paper is organized as follows: In Section 2 we define the resourced function space and we detail the construction of the initial solution of the corresponding domain equation. In Section 3 we define a similarity relation between the values of the standard function space and those of the resourced function space. In Section 4 we describe a standard and a resourced denotational semantics for a lazy $\lambda$-calculus, and we prove their equivalence. The last section is devoted to the conclusions and the outline of future work.

\section{A RESOURCED FUNCTION SPACE}

A partially ordered set $\left(D, \sqsubseteq_{D}\right)$ is a directed-complete partial order (dcpo) if every directed subset has a supremum. A complete partial order (cpo) is a dcpo with a least element. For simplicity a cpo is usually represented by just its set.

A function $f: D \rightarrow D^{\prime}$ between $\operatorname{cpos} D$ and $D^{\prime}$ is continuous if it maps directed sets to directed sets while preserving their suprema.

Following Scott's domain theory [6], the function space $\left[D \rightarrow D^{\prime}\right]$, being $(D, \sqsubseteq D)$ and $\left(D^{\prime}, \sqsubseteq_{D^{\prime}}\right)$ cpos, is defined as $\left[D \rightarrow D^{\prime}\right] \stackrel{\text { def }}{=}\left\{f \mid f: D \rightarrow D^{\prime}\right.$ is continuous $\}$, where the order is $f \sqsubseteq_{\left[D \rightarrow D^{\prime}\right]} g \stackrel{\text { def }}{=} \forall d \in D . f(d) \sqsubseteq_{D^{\prime}} g(d)$.

The lifted construction of a cpo $\left(D, \sqsubseteq_{D}\right)$ is defined as:

$$
\begin{array}{lll}
D_{\perp} & \stackrel{\text { def }}{=} & \{\perp\} \cup\{\lfloor d\rfloor \mid d \in D\}, \\
x \sqsubseteq_{\perp} y & \stackrel{\text { def }}{=} & x=\perp \vee\left(x=\lfloor d\rfloor \wedge y=\left\lfloor d^{\prime}\right\rfloor \wedge d \sqsubseteq_{D} d^{\prime}\right) .
\end{array}
$$

The function $\lfloor-\rfloor$ verifies that $\lfloor d\rfloor=\left\lfloor d^{\prime}\right\rfloor \Rightarrow d=d^{\prime}$ and $\perp \neq\lfloor d\rfloor$ for all $d, d^{\prime} \in D$.

Two functions, up ${ }_{D}: D \rightarrow D_{\perp}$ and $\operatorname{dn}_{D}: D_{\perp} \rightarrow D$, relate the cpo $D$ to its lifted version $D_{\perp}$ and viceversa:

$$
\begin{aligned}
& \operatorname{up}_{D}(d) \stackrel{\text { def }}{=}\lfloor d\rfloor, \quad \operatorname{dn}_{D}(\perp) \stackrel{\text { def }}{=} \perp_{D}, \\
& \operatorname{dn}_{D}(\lfloor d\rfloor) \stackrel{\text { def }}{=} d \text {. }
\end{aligned}
$$

They verify that $\operatorname{dn}_{D} \circ \mathrm{up}_{D}=i d_{D}$ and $i d_{D_{\perp}} \sqsubseteq \mathrm{up}_{D} \circ \mathrm{dn}_{D}$.

There are different ways of realising $\lfloor-\rfloor$, all of them leading to isomorphic constructions. In the following we will consider that $\lfloor d\rfloor=d$.

Let $f: D \rightarrow D^{\prime}$, it can be extended to $f_{\perp}: D_{\perp} \rightarrow D_{\perp}^{\prime}$ :

$$
\begin{array}{ll}
f_{\perp}(\perp) & \stackrel{\text { def }}{=} \perp, \text { and } \\
f_{\perp}\left(\operatorname{up}_{D}(d)\right) \stackrel{\text { def }}{=} & \operatorname{up}_{D^{\prime}}(f(d)) \text { for } d \in D .
\end{array}
$$

The construction of the initial solution of the domain equation $D=[D \rightarrow D]_{\perp}$ is detailed in [2]. The objective of the present section is to adapt this construction to obtain the initial solution for the domain equation $E=[[C \rightarrow$ $E] \rightarrow[C \rightarrow E]]_{\perp}$, being $C$ the least solution of the domain equation $C=C_{\perp}$. The elements of $C$ are represented as $\perp$, $S(\perp), S^{2}(\perp)=S(S(\perp)), \ldots, S^{n}(\perp), \ldots$, being $S^{\infty}(\perp)$ its limit element, where $S$ stands for a successor-like function. In the following, to simplify, we abbreviate $S^{\infty}(\perp)$ to $S^{\infty}$.

The initial solution for the domain equation $E=[[C \rightarrow$ $E] \rightarrow[C \rightarrow E]]_{\perp}$ is constructed by finite approximations. Each pair injection/projection between successive approximations constitutes an embedding.

\subsection{Embeddings}

Let $D$ and $D^{\prime}$ be cpos. An embedding of $D$ into $D^{\prime}$ is a pair of continuous maps $\langle i, j\rangle$ such that $D \stackrel{i}{\longmapsto} D^{\prime} \stackrel{j}{\rightarrow} D$, and $i \circ j \sqsubseteq i d_{D^{\prime}}$ and $j \circ i=i d_{D}$, where $\longmapsto$ and $\rightarrow$ stand for an injection and a projection respectively.

Given an embedding of $D$ into $D^{\prime}$, it is possible to build other embeddings between cpos constructed from $D$ and $D^{\prime}$.

Consider the composition application over cpos $X, Y$ and $Z$ with the following signature:

$$
\circ:[Y \rightarrow Z] \times[X \rightarrow Y] \rightarrow[X \rightarrow Z]
$$

Then, for any $f \in[Y \rightarrow Z]$ and $g \in[X \rightarrow Y]$, we can define the function sections:

$$
\begin{aligned}
& (f \circ):[X \rightarrow Y] \rightarrow[X \rightarrow Z] \text { and } \\
& (\circ g):[Y \rightarrow Z] \rightarrow[X \rightarrow Z],
\end{aligned}
$$

so that $(f \circ)(h) \stackrel{\text { def }}{=}(f \circ h)$ and $(\circ g)(h) \stackrel{\text { def }}{=}(h \circ g)$ for a function $h$ of the appropiate type in each case.

Lemma 1. Let $\langle i, j\rangle$ be an embedding of $D$ into $D^{\prime}$. For any cpo $X,\langle(i \circ),(j \circ)\rangle$ is an embedding of $[X \rightarrow D]$ into $\left[X \rightarrow D^{\prime}\right]$.

Proof. We have to show that $(j \circ) \circ(i \circ)=i d_{[X \rightarrow D]}$ and $(i \circ) \circ(j \circ) \sqsubseteq i d_{\left[X \rightarrow D^{\prime}\right]}$.

Let $f \in[X \rightarrow D]$, we have $(j \circ) \circ(i \circ)(f)=(j \circ) \circ(i \circ f)=$ $(j \circ i) \circ f=i d_{D} \circ f=f$, by associativity of $\circ$.

Similarly, if $g \in\left[X \rightarrow D^{\prime}\right]$ then $(i \circ) \circ(j \circ)(g)=(i \circ) \circ$ $(j \circ g)=(i \circ j) \circ g \sqsubseteq i d_{D^{\prime}} \circ g=g$. 
As a consequence, from an embedding of $D$ into $D^{\prime}$, an embedding between the resourced domains $[C \rightarrow D]$ and $\left[C \rightarrow D^{\prime}\right]$ can be built by choosing $X$ as the countable chain domain $C$ defined above.

Corollary 2. Let $\langle i, j\rangle$ be an embedding of $D$ into $D^{\prime}$. Then $\left\langle i^{C}, j^{C}\right\rangle$ is an embedding of $[C \rightarrow D]$ into $\left[C \rightarrow D^{\prime}\right]$, which is defined as $i^{C}(a)(c) \stackrel{\text { def }}{=} i(a(c))$ and $j^{C}(b)(c) \stackrel{\text { def }}{=} j(b(c))$, where $a \in[C \rightarrow D], b \in\left[C \rightarrow D^{\prime}\right]$ and $c \in C$.

Proof. Notice that $i^{C}=(i \circ)$ and $j^{C}=(j \circ)$; then we use Lemma 1.

Given an embedding between two cpos, an embedding between their lifted domains can be easily obtained.

Lemma 3. Let $\langle i, j\rangle$ be an embedding of $D$ into $D^{\prime}$. The pair $\left\langle i_{\perp}, j_{\perp}\right\rangle$ is an embedding of $D_{\perp}$ into $D_{\perp}^{\prime}$.

Proof. Trivial from the definition of $i_{\perp}$ and $j_{\perp}$.

Next we define how to build an embedding between function spaces from an embedding between their ground cpos.

Lemma 4. Let $\langle i, j\rangle$ be an embedding of $D$ into $D^{\prime}$. Then $\left\langle i^{\rightarrow}, j^{\rightarrow}\right\rangle$ is an embedding of $[D \rightarrow D]$ into $\left[D^{\prime} \rightarrow D^{\prime}\right]$, where:

$$
\begin{aligned}
& i \rightarrow \stackrel{\text { def }}{=}(i \circ) \circ(\circ j), \text { and } \\
& j \rightarrow \stackrel{\text { def }}{=}(j \circ) \circ(\circ i) .
\end{aligned}
$$

Proof. Since $\langle i, j\rangle$ is an embedding of $D$ into $D^{\prime}$, it is verified that $j \circ i=i d_{D}$ and $i \circ j \sqsubseteq i d_{D^{\prime}}$. We have to check that $j \rightarrow \circ i^{\rightarrow}=i d_{[D \rightarrow D]}$ and $i^{\rightarrow} \circ j \rightarrow \sqsubseteq i d_{\left[D^{\prime} \rightarrow D^{\prime}\right]}$. We use again the associativity of $\circ$.

Let $f \in[D \rightarrow D]$ and $g \in\left[D^{\prime} \rightarrow D^{\prime}\right]$ :

$$
\begin{aligned}
(i \rightarrow \circ j \rightarrow)(g) & =((i \circ) \circ(\circ j)) \circ((j \circ) \circ(\circ i))(g) \\
& ((i \circ) \circ(\circ j))(j \circ g \circ i) \\
& =i \circ(j \circ g \circ i) \circ j \\
& =(i \circ j) \circ g \circ(i \circ j) \\
& \sqsubseteq i d_{D}^{\prime} \circ g \circ i d_{D}^{\prime} \\
& =g .
\end{aligned}
$$

Similarly, it is proved that $\left(j \rightarrow \circ i^{\rightarrow}\right)(f)=f$.

Now we combine the two previous constructions to obtain an embedding between lifted function spaces.

Corollary 5. Let $\langle i, j\rangle$ be an embedding of $D$ into $D^{\prime}$. The pair $\left\langle i^{*}, j^{*}\right\rangle$, which is defined as $i^{*} \stackrel{\text { def }}{=}\left(i^{\rightarrow}\right)_{\perp}$ and $j^{*} \stackrel{\text { def }}{=}$ $\left(j^{\rightarrow}\right)_{\perp}$, is an embedding of $[D \rightarrow D]_{\perp}$ into $\left[D^{\prime} \rightarrow D^{\prime}\right]_{\perp}$.

Proof. Is a consequence of Lemmas 3 and 4 .

This corollary is equivalent to Lemma 4.1.1. given by Abramsky and Ong in [2]; although their definition of $\left\langle i^{*}, j^{*}\right\rangle$ is more cumbersome because the lifting and function space constructions are combined in a unique step.

These results also can be easily obtained by considering embeddings as Galois connections [8].

We are now ready to define an embedding of $[[C \rightarrow D] \rightarrow$ $[C \rightarrow D]]_{\perp}$ into $\left[\left[C \rightarrow D^{\prime}\right] \rightarrow\left[C \rightarrow D^{\prime}\right]\right]_{\perp}$. We start with an embedding $\langle i, j\rangle$ of $D$ into $D^{\prime}$; by Corollary 2 we obtain $\left\langle i^{C}, j^{C}\right\rangle$, an embedding of $[C \rightarrow D]$ into $\left[C \rightarrow D^{\prime}\right]$. Finally we get an embedding $\left\langle i^{C *}, j^{C *}\right\rangle$ of $[[C \rightarrow D] \rightarrow[C \rightarrow D]]_{\perp}$ into $\left[\left[C \rightarrow D^{\prime}\right] \rightarrow\left[C \rightarrow D^{\prime}\right]\right]_{\perp}$ by applying Corollary 5 (see Figure 1).

The embedding $\left\langle i^{C *}, j^{C *}\right\rangle$ is used in the next subsection to construct the initial solution of $E=[[C \rightarrow E] \rightarrow[C \rightarrow E]]_{\perp}$.

\subsection{Construction of the initial solution}

We represent the finite aproximations of $E$ by $\left\{E_{n}\right\}_{n \in \mathbb{N}}$, defined inductively as $E_{0} \stackrel{\text { def }}{=}\left\{\perp_{E_{0}}\right\}$ and $E_{n+1} \stackrel{\text { def }}{=}[[C \rightarrow$ $\left.\left.E_{n}\right] \rightarrow\left[C \rightarrow E_{n}\right]\right]_{\perp}$. Intuitively, $E_{n}$ contains the (resourced) functions that can be applied until level $n$ at most. Hence, $E_{0}$ contains only the bottom value, indicating that it cannot be applied. While $E_{1}$ has two elements, i. e., $E_{1}=$ $\left\{\perp_{E_{1}}, e_{1}\right\}$ where $e_{1}$ is the function that maps $a_{0}$ into $a_{0}$, being $A_{0}=\left[C \rightarrow E_{0}\right]=\left\{a_{0}\right\}$ such that $\forall n \in \mathbb{N}^{\infty}(=$ $\mathbb{N} \cup\{\infty\}) . a_{0}\left(S^{n}(\perp)\right)=\perp_{E_{0}}$. Therefore, $e_{1}$ can be applied in a first level (to $a_{0} \in A_{0}$ ) but the resulting value $\left(a_{0}\right)$ instantiated with any amount of resources produces $\perp_{E_{0}}$, which cannot be applied.

Let $\left\langle i_{0}, j_{0}\right\rangle$ be an embedding of $E_{0}$ into $E_{1}$, where $i_{0}$ and $j_{0}$ are defined as follows:

$$
\begin{array}{rlllllll}
i_{0}: E_{0} & \longrightarrow & E_{1} & j_{0} & : E_{1} & \longrightarrow & E_{0} \\
\perp_{E_{0}} & \mapsto & \perp_{E_{1}} & & \perp_{E_{1}} & \mapsto & \perp_{E_{0}} \\
& & & e_{1} & \mapsto & \perp_{E_{0}}
\end{array}
$$

The embeddings of $E_{n+1}$ into $E_{n+2}$, i. e. $E_{n+1} \stackrel{i_{n+1}}{\longmapsto} E_{n+2} \stackrel{j_{n+1}}{\rightarrow}$ $E_{n+1}$, are defined as $\left\langle i_{n+1}, j_{n+1}\right\rangle \stackrel{\text { def }}{=}\left\langle i_{n}^{C *}, j_{n}^{C *}\right\rangle$, that is,

$$
\begin{aligned}
i_{n+1}\left(e_{n+1}\right) & =i_{n}^{C *}\left(e_{n+1}\right)=\left(i_{n}^{C \rightarrow}\right)_{\perp}\left(e_{n+1}\right) \\
& =\left\{\begin{array}{lll}
\perp_{E_{n+2}} & \text { if } e_{n+1}=\perp_{E_{n+1}} \\
i_{n}^{C} \circ e_{n+1} \circ j_{n}^{C} & \text { if } e_{n+1} \neq \perp_{E_{n+1}}
\end{array}\right. \\
& =\left\{\begin{array}{lll}
\perp_{n+1}\left(e_{n+2}\right) & =j_{n}^{C *}\left(e_{n+2}\right)=\left(j_{n}^{C \rightarrow}\right)_{\perp}\left(e_{n+2}\right) \\
j_{n}^{C} \circ e_{n+2} \circ & i_{n}^{C} & \text { if } e_{n+2} \neq e_{n+2} \neq \perp_{E_{n+2}}
\end{array}\right.
\end{aligned}
$$

$\left\langle E_{n}, j_{n}\right\rangle_{n \in \mathbb{N}}$ forms an inverse system of cpos and we take its inverse limit [13] as $E$, i.e.,

$$
\begin{aligned}
E & =\lim _{\leftarrow}\left\langle E_{n}, j_{n}\right\rangle \\
& =\left\{\left\langle e_{n}: n \in \mathbb{N}\right\rangle \mid e_{n} \in E_{n} \wedge j_{n}\left(e_{n+1}\right)=e_{n}\right\} \subseteq \prod_{n \in \mathbb{N}} E_{n} .
\end{aligned}
$$

where the tuple $\left\langle e_{n}: n \in \mathbb{N}\right\rangle=\left\langle e_{0}, e_{1}, \ldots, e_{n}, \ldots\right\rangle$ represents an element $e \in E$ by its approximations in each layer.

The embeddings of $E_{n}$ into $E_{n+1}$ can be generalized to relate $E_{m}$ to $E_{n}$, for any $n, m \in \mathbb{N}^{\infty}$.

We define the functions $\phi_{m, n}^{E}: E_{m} \rightarrow E_{n}$ as follows:

$$
\begin{array}{ll}
m=n & \phi_{n, n}^{E} \stackrel{\text { def }}{=} i d_{E_{n}}, \\
m>n & \phi_{m, n}^{E} \stackrel{\text { def }}{=} \phi_{m-1, n}^{E} \circ j_{m-1}, \\
m<n & \phi_{m, n}^{E} \stackrel{\text { def }}{=} i_{n-1} \circ \phi_{m, n-1}^{E} .
\end{array}
$$

The $n$-projection $\phi_{\infty, n}^{E}: E \rightarrow E_{n}$ is defined as the limit of the projections $\phi_{m, n}^{E}$. For simplicity we write $\psi_{n}^{E}$ for $\phi_{\infty, n}^{E}$. Similarly, $\phi_{n}^{E}$ represents the $n$-injection $\phi_{n, \infty}^{E}: E_{n} \rightarrow E$. By construction $\left\langle\phi_{n}^{E}, \psi_{n}^{E}\right\rangle$ forms an embedding of $E_{n}$ into $E$.

Also, we view each $E_{n}$ as a subset of $E$, that is, we identify $\phi_{n}^{E}(x)$ with $x$, where $x \in E_{n}$; and for $e \in E, \psi_{n}^{E}(e)=e_{n} \in$ $E_{n}$. Thus, $E=\bigcup_{n \in \mathbb{N}} E_{n}$.

To illustrate our construction, we generate the three first approximations of $E$, i.e., $E_{0}, E_{1}$ and $E_{2}$, and we look into the corresponding embeddings.

As explained above, $E_{0}$ is the one-point domain represented by $\left\{\perp_{E_{0}}\right\}$, and $E_{1}=\left\{\perp_{E_{1}}, e_{1}\right\}$, where $e_{1}\left(a_{0}\right)=a_{0}$ and $\left\{a_{0}\right\}=A_{0}=\left[C \rightarrow E_{0}\right]$ is such that $a_{0}\left(S^{n}(\perp)\right)=\perp_{E_{0}}$ for all $n \in \mathbb{N}^{\infty}$. Now $E_{2}=\left[A_{1} \rightarrow A_{1}\right]_{\perp}$, where $A_{1}=\left[C \rightarrow E_{1}\right]$ (see Figure 2). The elements of $A_{1}$ are functions $a_{1, n}(n \in \mathbb{N})$ 


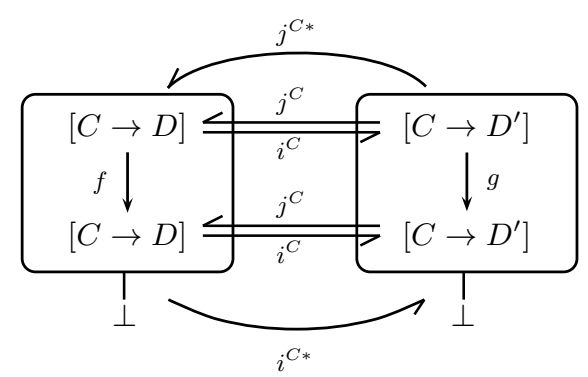

Figure 1: Embeddings on resourced domains

such that:

$$
a_{1, n}\left(S^{k}(\perp)\right)= \begin{cases}\perp_{E_{1}} & \text { if } k<n \\ e_{1} & \text { if } k \geq n\end{cases}
$$

We define $a_{1, \infty}$ as the function verifying that forall $n \in \mathbb{N}^{\infty}$ $a_{1, \infty}\left(S^{n}(\perp)\right)=\perp_{E_{1}}$, i.e., this is the least defined function in $A_{1}$. Notice that the most defined one is $a_{1,0}$, the constant function with $a_{1,0}\left(S^{n}(\perp)\right)=e_{1}$ forall $n \in \mathbb{N}^{\infty}$. Hence, we have an ordering in $A_{1}$ where $a_{1, \infty} \sqsubseteq a_{1, m} \sqsubseteq a_{1, n} \sqsubseteq a_{1,0}$ for all $m, n \in \mathbb{N}$ such that $n \leq m$.

Since $A_{1}=\left\{a_{1, n} \mid n \in \mathbb{N}^{\infty}\right\}$, any increasing $\omega$-chain in $A_{1}$ has as l.u.b. $a_{1, k}$ for some $k \in \mathbb{N}$, except for the constant chain $\left\{x_{n}=a_{1, \infty}\right\}_{n \in \mathbb{N}}$. Hence, we can characterize the continuous functions $e: A_{1} \rightarrow A_{1}$ as those that satisfy the following property:

$$
n \leq m \Rightarrow l \leq k, \text { for } e\left(a_{1, m}\right)=a_{1, k} \text { and } e\left(a_{1, n}\right)=a_{1, l}
$$

Summarizing,

$$
E_{2}=\left\{\perp_{E_{2}}\right\} \cup\left\{e:\left\{a_{1, n}\right\}_{n \in \mathbb{N}^{\infty}} \rightarrow\left\{a_{1, n}\right\}_{n \in \mathbb{N}^{\infty}} \mid e \text { sat.(1) }\right\} .
$$

The representation of the embeddings $\left\langle i_{0}, j_{0}\right\rangle$ of $E_{0}$ into $E_{1}$ and $\left\langle i_{1}, j_{1}\right\rangle$ of $E_{1}$ into $E_{2}$ is shown in Figure 3 . For $E_{2}$ we only highlight the constant functions $e_{2, \infty}$ and $e_{2,0}$ representing the two extremes of $\left[A_{1} \rightarrow A_{1}\right]$, i.e., for all $n \in \mathbb{N}^{\infty}$

$$
e_{2, \infty}\left(a_{1, n}\right)=a_{1, \infty} \quad \text { and } \quad e_{2,0}\left(a_{1, n}\right)=a_{1,0} .
$$

\subsection{Application operations}

Application operations are defined in each domain $E_{n}$ as $\operatorname{Ap}_{E_{n}}^{\perp}: E_{n+1} \times A_{n} \times C \rightarrow E_{n}$,

$$
\operatorname{Ap}_{E_{n}}\left(e_{n+1}, a_{n}, c\right) \stackrel{\text { def }}{=} \begin{cases}\perp_{E_{n}} & \text { if } e_{n+1}=\perp_{E_{n+1}} \\ e_{n+1}\left(a_{n}\right)(c) & \text { if } e_{n+1} \neq \perp_{E_{n+1}}\end{cases}
$$

$\psi_{n}^{E}$ denotes the $n$-projection defined in Section 2.2 for the domain $E$. From the embedding $\left\langle\phi_{n}^{E}, \psi_{n}^{E}\right\rangle$ of $E_{n}$ into $E$ and using Corollary 2, we obtain the embedding $\left\langle\left(\phi_{n}^{E}\right)^{C},\left(\psi_{n}^{E}\right)^{C}\right\rangle$ of $\left[C \rightarrow E_{n}\right]$ into $[C \rightarrow E]$, so that $\psi_{n}^{[C \rightarrow E]}$ stands for $\left(\psi_{n}^{E}\right)^{C}$. Application operation in $E$ is $\mathrm{AP}_{E}^{\perp}: E \times[C \rightarrow E] \times C \rightarrow E$,

$$
\operatorname{AP}_{E}^{\perp}(e, a, c) \stackrel{\text { def }}{=} \bigsqcup_{n \in \mathbb{N}} \operatorname{Ap}_{E_{n}}^{\perp}\left(\psi_{n+1}^{E}(e), \psi_{n}^{[C \rightarrow E]}(a), c\right) .
$$

In the following, we use $e(a)(c)$ for both $\operatorname{Ap}_{E_{n}}^{\perp}(e, a, c)$ and $\operatorname{AP}_{E} \frac{\perp}{E}(e, a, c)$; from the context it should be clear which is the correct one. Notice that

$$
\psi_{n}^{E}(e(a)(c))=\psi_{n+1}^{E}(e)\left(\psi_{n}^{[C \rightarrow E]}(a)\right)(c) .
$$

Analogously, from the definition of the application operation in the standard lifted function space $D$ described in [2],

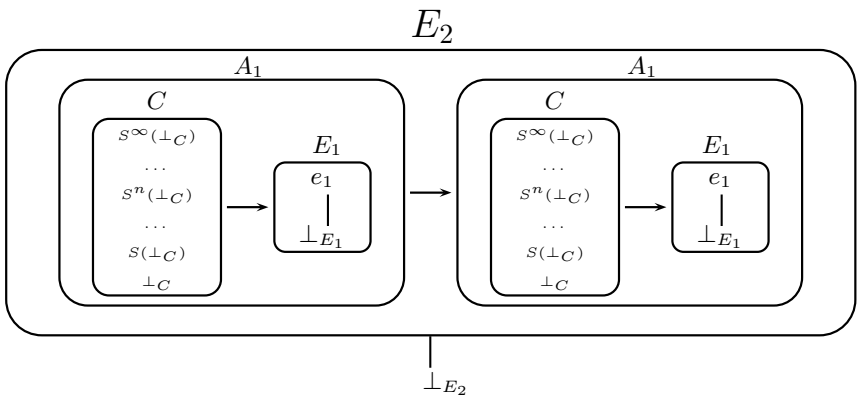

Figure 2: The domain $E_{2}$

where $D$ is the initial solution of $D=[D \rightarrow D]_{\perp}$ and this initial solution can be described as an inverse limit similarly to $\mathrm{E}$, it can be observed that

$$
\psi_{n}^{D}\left(d\left(d^{\prime}\right)\right)=\psi_{n+1}^{D}(d)\left(\psi_{n}^{D}\left(d^{\prime}\right)\right) .
$$

\section{SIMILARITY}

In this section we define a relation between the standard lifted function domain $D=[D \rightarrow D]_{\perp}$ and the resourced domain $E=[[C \rightarrow E] \rightarrow[C \rightarrow E]]_{\perp}$ constructed in the previous section. Our relation is inspired by the applicative bisimulation defined by Abramsky in [1], so that functions $d \in D$ and $e \in E$ are considered to be similar if $d$ and $e$ have a similar applicative behaviour when infinite resources are available for $e$, i.e., they produce similar values when applied to similar arguments.

To formalize this circular definition we resort to a layered construction, that is, we inductively define a sequence of relations $\left\{\triangleleft \triangleright_{n} \subseteq D_{n} \times E_{n}\right\}_{n \in \mathbb{N}}$.

DEFINITION 1 (n-SIMILARITY). A family of $n$-similarity relations between elements of $D_{n}$ and $E_{n}$, for $n \in \mathbb{N}$, is defined as follows:

- 0-similarity, $\triangleleft \triangleright_{0}: \perp_{D_{0}} \triangleleft \triangleright_{0} \quad \perp_{E_{0}}$.

- $n+1$-similarity, $\triangleleft \triangleright_{n+1}$, is the least relation in $D_{n+1} \times$ $E_{n+1}$ that verifies:

(1) $\perp_{D_{n+1}} \triangleleft \triangleright_{n+1} \perp_{E_{n+1}}$ and,

(2) let $d \in D_{n+1}$ and $e \in E_{n+1}$ such that $d \neq \perp_{D_{n+1}}$ and $e \neq \perp_{E_{n+1}}$, then $d \triangleleft \triangleright_{n+1} e$ if for any $d^{\prime} \in D_{n}$ and for any $a^{\prime} \in A_{n}, d^{\prime} \triangleleft \triangleright_{n} a^{\prime}\left(S^{\infty}\right) \Rightarrow d\left(d^{\prime}\right) \triangleleft \triangleright_{n} e\left(a^{\prime}\right)\left(S^{\infty}\right)$.

For a better understanding of this definition, we show the three first layers (Figure 4):

- 0-similarity $\left(\triangleleft \triangleright_{0}\right): D_{0}=\left\{\perp_{D_{0}}\right\}$ and $E_{0}=\left\{\perp_{E_{0}}\right\}$, so that the relation reduces to $\perp_{D_{0}} \triangleleft \triangle_{0} \perp_{E_{0}}$.

- 1-similarity $\left(\triangleleft \triangleright_{1}\right): D_{1}=\left\{\perp_{D_{1}}, d_{1}\right\}$, where $d_{1} \in\left[D_{0} \rightarrow\right.$ $\left.D_{0}\right]$ is the function mapping $\perp_{D_{0}}$ into $\perp_{D_{0}}$, and recall that $E_{1}=\left\{\perp_{E_{1}}, e_{1}\right\}$ with $e_{1}\left(a_{0}\right)=a_{0} . \perp_{D_{1}} \triangleleft \triangleright_{1} \quad \perp_{E_{1}}$, by definition. Now let $d^{\prime} \in D_{0}$ and $a^{\prime} \in A_{0}=\left\{a_{0} \mid \forall n \in\right.$ $\left.\mathbb{N}^{\infty} \cdot a_{0}\left(S^{n}(\perp)\right)=\perp_{E_{0}}\right\}$, it must be that $d^{\prime}=\perp_{D_{0}}$ and $a^{\prime}=$ $a_{0}$. Hence, we have that $\perp_{D_{0}}=d^{\prime} \triangleleft \triangleright_{0} \quad a^{\prime}\left(S^{\infty}\right)=\perp_{E_{0}}$. Moreover, $d_{1}\left(d^{\prime}\right)=\perp_{D_{0}} \quad \triangleleft \triangleright_{0} \quad \perp_{E_{0}}=a_{0}\left(S^{\infty}\right)=e_{1}\left(a_{0}\right)\left(S^{\infty}\right)=$ $e_{1}\left(a^{\prime}\right)\left(S^{\infty}\right)$. Thus, $d_{1} \triangleleft \triangleright_{1} e_{1}$.

- 2-similarity $\left(\triangleleft \triangleright_{2}\right): D_{2}=\left\{\perp_{D_{2}}, d_{2,1}, d_{2,2}, d_{2,3}\right\}$, where the functions $d_{2,1}, d_{2,2}$ and $d_{2,3}$ are defined as follows:

$$
\begin{array}{lll}
d_{2,1}\left(\perp_{D_{1}}\right)=\perp_{D_{1}} & d_{2,2}\left(\perp_{D_{1}}\right)=\perp_{D_{1}} & d_{2,3}\left(\perp_{D_{1}}\right)=d_{1} \\
d_{2,1}\left(d_{1}\right)=\perp_{D_{1}} & d_{2,2}\left(d_{1}\right)=d_{1} & d_{2,3}\left(d_{1}\right)=d_{1}
\end{array}
$$




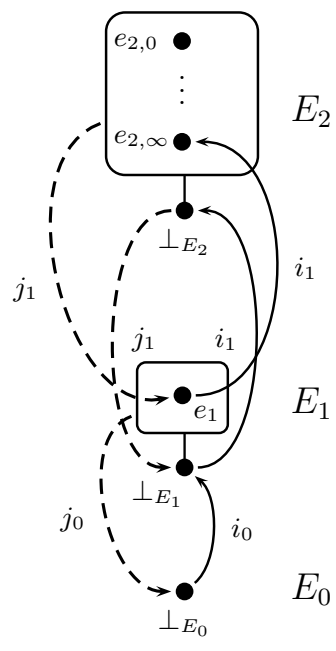

Figure 3: First steps of the construction of $E$

We have shown in Subsection 2.2 that

$E_{2}=\left\{\perp_{E_{2}}\right\} \cup\left\{e:\left\{a_{1, n}\right\}_{n \in \mathbb{N}^{\infty}} \rightarrow\left\{a_{1, n}\right\}_{n \in \mathbb{N}^{\infty}} \mid e\right.$ sat. (1) $\}$.

By definition, $\perp_{D_{2}} \triangleleft \triangleright_{2} \perp_{E_{2}}$.

Let $d^{\prime} \in D_{1}$ and $a^{\prime} \in A_{1}=\left[C \rightarrow E_{1}\right]$ such that $d^{\prime} \triangleleft \triangleright_{1} a^{\prime}\left(S^{\infty}\right)$. Consequently, either $d^{\prime}=\perp_{D_{1}}$ and $a^{\prime}=a_{1, \infty}$ (and hence $\left.a^{\prime}\left(S^{\infty}\right)=\perp_{E_{1}}\right)$, or $d^{\prime}=d_{1}$ and $a^{\prime}=a_{1, k}$ for some $k \in \mathbb{N}$ (notice that $\forall n \in \mathbb{N} \cdot a_{1, n}\left(S^{\infty}\right)=e_{1}$ ). Therefore, the functions in $A_{1}$ can be partitioned into two classes: $\left[a_{1, \infty}\right]$ is the class of functions returning $\perp_{E_{1}}$ when applied to $S^{\infty}$; whereas the functions in $\left[a_{1,0}\right]$ return $e_{1}$ when infinite resources are provided, i.e., $\left[a_{1,0}\right]=\left\{a_{1, n} \mid a_{1, n}\left(S^{\infty}\right)=e_{1}\right\}$. Notice that the first class contains a unique element, that is, $\left[a_{1, \infty}\right]=\left\{a_{1, \infty}\right\}$. But it could be proved that 2 -similarity requires that the classes in $A_{1}$ are preserved, i.e., for any $e \in E_{2}$ there exists $d \in D_{2}$ such that $d \triangleleft \triangleright_{2} e$ only if

$\forall a, a^{\prime} \in A_{1} \cdot\left(a\left(S^{\infty}\right)=a^{\prime}\left(S^{\infty}\right) \Rightarrow e(a)\left(S^{\infty}\right)=e\left(a^{\prime}\right)\left(S^{\infty}\right)\right)$.

Therefore, $E_{2}=\left\{\perp_{E_{2}}\right\} \cup\left[e_{2,1}\right] \cup\left[e_{2,2}\right] \cup\left[e_{2,3}\right] \cup E_{2}^{\prime}$. where

$$
\begin{aligned}
& {\left[e_{2,1}\right]=\left\{e \in E_{2} \mid \forall a \in A_{1} . e(a) \in\left[a_{1, \infty}\right]\right\},} \\
& {\left[e_{2,2}\right]=\left\{e \in E_{2} \mid \forall a \in A_{1} .\left(a \in\left[a_{1, \infty}\right] \Rightarrow e(a) \in\left[a_{1, \infty}\right]\right)\right.} \\
& \left.\qquad e^{\wedge}\left(a \in\left[a_{1,0}\right] \Rightarrow e(a) \in\left[a_{1,0}\right]\right)\right\}, \\
& {\left[e_{2,3}\right]=\left\{e \in E_{2} \mid \forall a \in A_{1} . e(a) \in\left[a_{1,0}\right]\right\} .}
\end{aligned}
$$

Hence, we have that $\perp_{D_{2}} \triangleleft \triangleright_{2} \perp_{E_{2}}, \forall e \in\left[e_{2,1}\right] . d_{2,1} \triangleleft \triangleright_{2} e$, $\forall e \in\left[e_{2,2}\right] . d_{2,2} \triangleleft \triangleright_{2} e, \forall e \in\left[e_{2,3}\right] . d_{2,3} \triangleleft \triangleright_{2} e$, and $E_{2}^{\prime}$ contains the elements of $E_{2}$ for which there is no similar element in $D_{2}$. This is graphically represented in Figure 4 .

Next we prove some useful properties of the $n$-similarity, e.g., that it preserves undefinedness:

Lemma 6. Let $n \in \mathbb{N}, d \in D_{n}$ and $e \in E_{n}$. If $d \triangleleft \triangleright_{n} e$ then either $\left(d=\perp_{D_{n}} \wedge e=\perp_{E_{n}}\right)$ or $\left(d \neq \perp_{D_{n}} \wedge e \neq \perp_{E_{n}}\right)$.

PROOF. Trivial by the definition of $\triangleleft \triangleright_{n}$.

In the following lemma $\left\langle i_{n}^{D}, j_{n}^{D}\right\rangle$ stands for the embedding of $D_{n}$ into $D_{n+1}$ [1], $\left\langle i_{n}^{E}, j_{n}^{E}\right\rangle$ for the embedding of $E_{n}$ into $E_{n+1}$, and $\left\langle i_{n}^{C}, j_{n}^{C}\right\rangle$ for the embedding of $\left[C \rightarrow E_{n}\right]$ into $[C \rightarrow$ $\left.E_{n+1}\right]$. The lemma states that the injections and projections of these embeddings preserve the similarity relation.

Lemma 7. Let $n \in \mathbb{N}, d \in D_{n+1}, e \in E_{n+1}, a \in A_{n+1}$, $d^{\prime} \in D_{n}, e^{\prime} \in E_{n}$ and $a^{\prime} \in A_{n}$ :
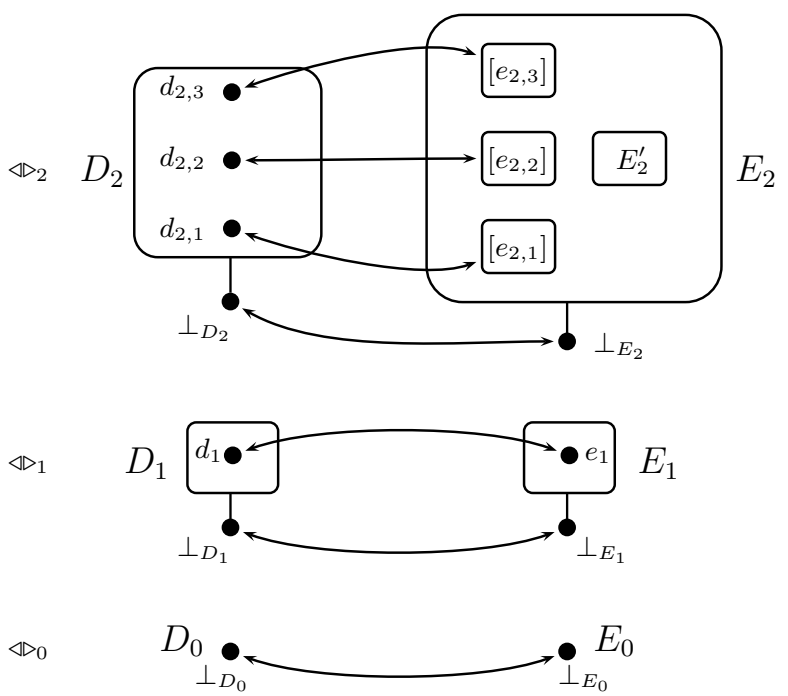

Figure 4: 0,1,2-similarity
1. $d \triangleleft \triangleright_{n+1} e \Rightarrow j_{n}^{D}(d) \triangleleft \triangleright_{n} j_{n}^{E}(e)$,
2. $d^{\prime} \triangleleft \triangleright_{n} e^{\prime} \Rightarrow i_{n}^{D}\left(d^{\prime}\right) \triangleleft \triangleright_{n+1} i_{n}^{E}\left(e^{\prime}\right)$,
3. $d \triangleleft \triangleright_{n+1} a\left(S^{\infty}\right) \Rightarrow j_{n}^{D}(d) \triangleleft \triangleright_{n} j_{n}^{C}(a)\left(S^{\infty}\right)$, and
4. $d^{\prime} \triangleleft \triangleright_{n} a^{\prime}\left(S^{\infty}\right) \Rightarrow i_{n}^{D}\left(d^{\prime}\right) \triangleleft \triangleright_{n+1} i_{n}^{C}\left(a^{\prime}\right)\left(S^{\infty}\right)$.

Proof. By induction on $n$.

- $n=0$ : By definition, $j_{0}^{D}(d)=\perp_{D_{0}}$ for any $d \in D_{1}, j_{0}^{E}(e)=\perp_{E_{1}}$ for any $e \in E_{1}$, and $j_{0}^{C}(a)\left(S^{\infty}\right)=\perp_{E_{0}}$ for any $a \in A_{1}$. Therefore, $j_{0}^{D}(d) \triangleleft \triangleright_{0} j_{0}^{E}(e)$ and $j_{0}^{D}(d) \triangleleft \triangleright_{0} j_{0}^{E}(a)\left(S^{\infty}\right)$.

Likewise, $i_{0}^{D}\left(d^{\prime}\right)=\perp_{D_{1}}$ for any $d^{\prime} \in D_{0}, i_{0}^{E}\left(e^{\prime}\right)=\perp_{E_{1}}$ for any $e^{\prime} \in E_{0}$, and $i_{0}^{C}\left(a^{\prime}\right)\left(S^{\infty}\right)=\perp_{E_{1}}$ for any $a^{\prime} \in A_{0}$. Therefore, $i_{0}^{D}\left(d^{\prime}\right) \triangleleft \triangleright_{1} i_{0}^{E}\left(e^{\prime}\right)$ and $i_{0}^{D}\left(d^{\prime}\right) \triangleleft \triangleright_{1} i_{0}^{C}\left(a^{\prime}\right)\left(S^{\infty}\right)$.

- $n>0$ :

(1). We assume $d \triangleleft \triangleright_{n+1} e$, hence (by Lemma 6) either $d=\perp_{D_{n+1}}$ and $e=\perp_{E_{n+1}}$, or $d \neq \perp_{D_{n+1}}$ and $e \neq \perp_{E_{n+1}}$.

The first case is trivial by the definiton of $j_{n}^{D}$ and $j_{n}^{E}$.

To prove the second case, let $d^{\prime \prime} \in D_{n-1}$, and $a^{\prime \prime} \in A_{n-1}$ such that $d^{\prime \prime} \triangleleft \triangleright_{n-1} a^{\prime \prime}\left(S^{\infty}\right)$. By induction hypothesis (4), we have that $i_{n-1}^{D}\left(d^{\prime \prime}\right) \triangleleft \triangleright_{n} i_{n-1}^{C}\left(a^{\prime \prime}\right)\left(S^{\infty}\right)$. As $d \triangleleft \triangleright_{n+1} e$, it must be that $d\left(i_{n-1}^{D}\left(d^{\prime \prime}\right)\right) \triangleleft \triangleright_{n} e\left(i_{n-1}^{C}\left(a^{\prime \prime}\right)\right)\left(S^{\infty}\right)$.

Then, by induction hypothesis (3),

$$
j_{n-1}^{D}\left(d\left(i_{n-1}^{D}\left(d^{\prime \prime}\right)\right)\right) \triangleleft \triangleright_{n-1} j_{n-1}^{C}\left(e\left(i_{n-1}^{C}\left(a^{\prime \prime}\right)\right)\right)\left(S^{\infty}\right),
$$

and by definition of $j_{n}^{D}$ and $j_{n}^{E}$ we have that

$$
j_{n}^{D}(d)\left(d^{\prime \prime}\right) \triangleleft \triangleright_{n-1} j_{n}^{E}(e)\left(a^{\prime \prime}\right)\left(S^{\infty}\right) .
$$

Therefore we have proved that $j_{n}^{D}(d) \triangleleft \triangleright_{n} j_{n}^{E}(e)$.

(4). We assume $d^{\prime} \triangleleft \triangleright_{n} a^{\prime}\left(S^{\infty}\right)$, thus (by Lemma 6) either $d^{\prime}=\perp_{D_{n}}$ and $a^{\prime}\left(S^{\infty}\right)=\perp_{E_{n}}$, or $d^{\prime} \neq \perp_{D_{n}}$ and $a^{\prime}\left(S^{\infty}\right) \neq \perp_{E_{n}}$.

The first case is trivial by the definition of $i_{n}^{D}$ and $i_{n}^{C}$.

For the second case, let $d^{\prime \prime} \in D_{n}$ and $a^{\prime \prime} \in A_{n}$ such that $d^{\prime \prime} \triangleleft \triangleright_{n} a^{\prime \prime}\left(S^{\infty}\right)$. By induction hypothesis (3),

$$
j_{n-1}^{D}\left(d^{\prime \prime}\right) \triangleleft \triangleright_{n-1} j_{n-1}^{C}\left(a^{\prime \prime}\right)\left(S^{\infty}\right) .
$$

As $d^{\prime} \triangleleft \triangleright_{n} a^{\prime}\left(S^{\infty}\right)$, it must be that

$$
d^{\prime}\left(j_{n-1}^{D}\left(d^{\prime \prime}\right)\right) \triangleleft \triangleright_{n-1} a^{\prime}\left(S^{\infty}\right)\left(j_{n-1}^{C}\left(a^{\prime \prime}\right)\right)\left(S^{\infty}\right) .
$$

Then, by induction hypothesis (4), we have that

$$
i_{n-1}^{D}\left(d^{\prime}\left(j_{n-1}^{D}\left(d^{\prime \prime}\right)\right)\right) \triangleleft \triangleright_{n} i_{n-1}^{C}\left(a^{\prime}\left(S^{\infty}\right)\left(j_{n-1}^{C}\left(a^{\prime \prime}\right)\right)\right)\left(S^{\infty}\right),
$$


and by definition of $i_{n}^{D}$ and $i_{n}^{C}$ we have that

$$
i_{n}^{D}(d)\left(d^{\prime \prime}\right) \triangleleft \triangleright_{n} i_{n}^{C}\left(a^{\prime}\left(S^{\infty}\right)\right)\left(a^{\prime \prime}\right)\left(S^{\infty}\right) .
$$

Therefore we have proved that $i_{n}^{D}\left(d^{\prime}\right) \triangleleft \triangleright_{n+1} i_{n}^{C}\left(a^{\prime}\right)\left(S^{\infty}\right)$. Proofs for (2) and (3) are similar to those for (1) and (4).

The previous lemma enables to pass the similarity relation up and down through the approximations of $D$ and $E$. We define a similarity relation between functions in $D$ and $E$.

Definition 2 (Similarity). $\triangleleft \triangleright$ is defined as the least relation in $D \times E$ that verifies that for each $d \in D$ and $e \in E$, $d \triangleleft \triangleright$ e if $\forall n \in \mathbb{N} . \psi_{n}^{D}(d) \triangleleft \triangleright_{n} \psi_{n}^{E}(e)$.

Similarity preserves undefinedness.

Corollary 8. Let $d \in D$ and $e \in E$. If $d \triangleleft \triangleright$ e then either $\left(d=\perp_{D} \wedge e=\perp_{E}\right)$ or $\left(d \neq \perp_{D} \wedge e \neq \perp_{E}\right)$.

Proof. Is a corollary of Lemma 6.

We give an alternative characterization for $\triangleleft \triangleright$ which is more convenient for writing proofs involving $\triangleleft \triangleright$.

Proposition 9. Let $d \in D, e \in E . d \triangleleft \triangleright e$ if and only if:

- $\left(d=\perp_{D} \wedge e=\perp_{E}\right)$, or

- $\left(d \neq \perp_{D} \wedge e \neq \perp_{E}\right) \wedge \forall d^{\prime} \in D . \forall a^{\prime} \in[C \rightarrow E]$.

$$
d^{\prime} \triangleleft \triangleright a^{\prime}\left(S^{\infty}\right) \Rightarrow d\left(d^{\prime}\right) \triangleleft \triangleright e\left(a^{\prime}\right)\left(S^{\infty}\right) .
$$

Proof. To simplify the notation we write $r_{n}$ for $\psi_{n}^{R}(r)$ where $n \in \mathbb{N}, r \in R$ and $R \in\{D, E,[C \rightarrow E]\}$.

(if) We first prove that if $d$ and $e$ verify the property then they are similar.

- Case 1: $d=\perp_{D}$ and $e=\perp_{E}$.

We have that $\left(\perp_{D}\right)_{n}=\perp_{D_{n}}$ and $\left(\perp_{E}\right)_{n}=\perp_{E_{n}}$ for all $n \in \mathbb{N}$. By definiton of $\triangleleft \triangleright_{n},\left(\perp_{D}\right)_{n}=\perp_{D_{n}} \triangleleft \triangleright_{n} \perp_{E_{n}}=\left(\perp_{E}\right)_{n}$, for any $n \in \mathbb{N}$. Therefore, $d \triangleleft \triangleright e$.

- Case 2: $d \neq \perp_{D}$ and $e \neq \perp_{E}$.

By assumption, $d^{\prime \prime} \triangleleft \triangleright a^{\prime \prime}\left(S^{\infty}\right) \Rightarrow d\left(d^{\prime \prime}\right) \triangleleft \triangleright e\left(a^{\prime \prime}\right)\left(S^{\infty}\right)$, for any $d^{\prime \prime} \in D$ and any $a^{\prime \prime} \in[C \rightarrow E]$.

By definition of $\triangleleft \triangleright$, we can assure that if $d\left(d^{\prime \prime}\right) \triangleleft \triangleright e\left(a^{\prime \prime}\right)\left(S^{\infty}\right)$, then $\forall m \in \mathbb{N} .\left(d\left(d^{\prime \prime}\right)\right)_{m} \triangleleft \triangleright_{m} \quad\left(e\left(a^{\prime \prime}\right)\left(S^{\infty}\right)\right)_{m}$. Then, by the equations (2) and (3) in Section 2.3, we have that

$\forall m \in \mathbb{N} . d_{m+1}\left(d_{m}^{\prime \prime}\right) \triangleleft \triangleright_{m} e_{m+1}\left(a_{m}^{\prime \prime}\right)\left(S^{\infty}\right)$.

Consequently, for any $d^{\prime \prime} \in D$ and any $a^{\prime \prime} \in[C \rightarrow E]$,

$$
d^{\prime \prime} \triangleleft \triangleright a^{\prime \prime}\left(S^{\infty}\right) \Rightarrow \forall m \in \mathbb{N} . d_{m+1}\left(d_{m}^{\prime}\right) \triangleleft \triangleright_{m} e_{m+1}\left(a_{m}^{\prime}\right)\left(S^{\infty}\right) .
$$

Now we have to prove that $d \triangleleft \triangleright$ e, i.e., $\forall n \in \mathbb{N}$. $d_{n} \triangleleft \triangleright_{n} e_{n}$. But $d_{0}=\perp_{D_{0}}$ and $e_{0}=\perp_{E_{0}}$ and, by definition, $\perp_{D_{0}} \triangleleft \triangleright_{0} \perp_{E_{0}}$. Thus, we have to check that $\forall n>0 . d_{n} \triangleleft \triangleright_{n} e_{n}$, or equivalently, that $\forall n \in \mathbb{N} . d_{n+1} \triangleleft \triangleright_{n+1} \quad e_{n+1}$.

That is, it must be verified that for any $d^{\prime} \in D_{n}$ and for any $a^{\prime} \in A_{n} d^{\prime} \triangleleft \triangleright_{n} a^{\prime}\left(S^{\infty}\right) \Rightarrow d_{n+1}\left(d^{\prime}\right) \triangleleft \triangleright_{n} e_{n+1}\left(a^{\prime}\right)\left(S^{\infty}\right)$.

Let $d^{\prime} \in D_{n}$, we construct a tuple

$$
\overline{d^{\prime}}=\left\langle d_{0}^{\prime}, d_{1}^{\prime}, \ldots, d_{n-1}^{\prime}, d^{\prime}, d_{n+1}^{\prime} \ldots\right\rangle
$$

such that $d_{k}^{\prime}=j_{k}^{D}\left(d_{k+1}^{\prime}\right)$ for $k<n$ and $d_{k}^{\prime}=i_{k-1}\left(d_{k-1}^{\prime}\right)$ for $k>n$. Therefore, $\overline{d^{\prime}} \in D$.

Likewise, let $a^{\prime} \in A_{n}=\left[C \rightarrow E_{n}\right]$, we construct

$$
\overline{a^{\prime}}=\left\langle a_{0}^{\prime}, a_{1}^{\prime}, \ldots, a_{n-1}^{\prime}, a^{\prime}, a_{n+1} \ldots\right\rangle \in[C \rightarrow E] .
$$

By Lemma 7 we can assure that $\overline{d^{\prime}} \triangleleft \triangleright \overline{a^{\prime}}\left(S^{\infty}\right)$ whenever $d^{\prime} \triangleleft \triangleright_{n} a^{\prime}\left(S^{\infty}\right)$. Then, by (4) we have that

$$
\forall m \in \mathbb{N} . d_{m+1}\left({\overline{d^{\prime}}}_{m}\right) \triangleleft \triangleright_{m} e_{m+1}\left({\overline{a^{\prime}}}_{m}\right)\left(S^{\infty}\right),
$$

and particularly: $d_{n+1}\left(d^{\prime}\right) \triangleleft \triangleright_{n} e_{n+1}\left(a^{\prime}\right)\left(S^{\infty}\right)$.

(only if) Let us prove that if $d \triangleleft \triangleright e$ then $d$ and $e$ satisfy the property. By Corollary 8 we only have two cases:

- Case 1: $d=\perp_{D}$ and $e=\perp_{E}$. Trivial.

- Case 2: $d \neq \perp_{D}$ and $e \neq \perp_{E}$.

Let $d^{\prime} \in D$ and $a^{\prime} \in[C \rightarrow E]$ such that $d^{\prime} \triangleleft \triangleright a^{\prime}\left(S^{\infty}\right)$.

We have to prove that $d\left(d^{\prime}\right) \triangleleft \triangleright e\left(a^{\prime}\right)\left(S^{\infty}\right)$, that is,

$\forall n \in \mathbb{N} .\left(d\left(d^{\prime}\right)\right)_{n} \triangleleft \triangleright_{n}\left(e\left(a^{\prime}\right)\left(S^{\infty}\right)\right)_{n}$.

For $n=0$ this is trivial. Now consider $n>0$; by hypothesis, $d \triangleleft \triangleright e$ and $d^{\prime} \triangleleft \triangleright a^{\prime}\left(S^{\infty}\right)$, therefore, by definition of $\triangleleft \triangleright$ we have that $\forall m \in \mathbb{N} . d_{m} \triangleleft \triangleright_{m} e_{m} \wedge d_{m}^{\prime} \triangleleft \triangleright_{m} \quad a_{m}^{\prime}\left(S^{\infty}\right)$.

Particularly: $d_{n+1} \triangleleft \triangleright_{n+1} \quad e_{n+1}$ and $d_{n}^{\prime} \triangleleft \triangleright_{n} a_{n}^{\prime}\left(S^{\infty}\right)$.

Consequently, $d_{n+1}\left(d_{n}^{\prime}\right) \triangleleft \triangleright_{n} e_{n+1}\left(a_{n}^{\prime}\right)\left(S^{\infty}\right)$, and therefore, $d\left(d^{\prime}\right)_{n} \triangleleft \triangleright_{n}\left(e\left(a^{\prime}\right)\left(S^{\infty}\right)\right)_{n}$, by (2) and (3) in Section 2.3.

\section{A DENOTATIONAL SEMANTICS FOR A LAZY $\lambda$-CALCULUS}

The language described in [7] is a normalised $\lambda$-calculus extended with recursive lets. The restricted syntax is given in Figure 5, where all bound variables are distinct, and applications are of an expression to a variable.

We consider a heap $\Gamma$ as a finite partial function from variables to expressions, that is, Heap $\in \mathcal{P}_{f}(\operatorname{Var} \times$ Exp $)$, with all the variables different pairwise.

\subsection{A standard denotational semantics}

To define a denotational semantics for this calculus, a domain of values and environments to associate values to the variables are needed.

An environment maps variables into values,

$$
\rho \in E n v=\text { Var } \rightarrow \text { Value },
$$

where Value is some appropriate domain containing at least a lifted version of its own function space, i.e.,

$$
v \in \text { Value }=[\text { Value } \rightarrow \text { Value }]_{\perp} .
$$

Notice that Value corresponds to the standard lifted domain $D$ described in [2].

An ordering is defined on environments, such that if $\rho$ is less or equal than $\rho^{\prime}$ then $\rho^{\prime}$ may bind more variables than $\rho$, but otherwise is equal to $\rho$. Formally, let $\rho, \rho^{\prime} \in E n v$ be environments, $\rho$ is less or equal to $\rho^{\prime}$ (denoted as $\rho \leq \rho^{\prime}$ ) iff $\forall x . \rho x \neq \perp \Rightarrow \rho x=\rho^{\prime} x$.

For the language described by the syntax in Figure 5, Launchbury defines a denotational semantics in [7]. The semantic function is $\llbracket-\rrbracket:$ Exp $\rightarrow$ Env $\rightarrow$ Value, which is given in Figure 6.

The function $\{\{-\}:$ Heap $\rightarrow$ Env $\rightarrow$ Env should be thought as an environment modifier defined as follows:

$$
\left\{\left(x_{i} \mapsto e_{i}\right)_{i=1}^{n}\right\} \rho=\mu \rho^{\prime} . \rho \sqcup\left(x_{i} \mapsto \llbracket e_{i} \rrbracket_{\rho^{\prime}}\right)_{i=1}^{n},
$$

where $\mu$ stands for the least fixed point operator and $\sqcup$ for

$$
\left(\rho \sqcup\left(x \mapsto \llbracket e \rrbracket_{\rho^{\prime}}\right)\right) y= \begin{cases}\rho(y) & \text { if } y \neq x \\ \llbracket e \rrbracket_{\rho^{\prime}} & \text { if } y=x\end{cases}
$$

This definition only makes sense on environments $\rho$ which are consistent with a heap $\Gamma$ (i.e., if $\rho$ and $\Gamma$ bind the same variables, then they are bound to values for which an upper bound exists). This consistency is guaranteed in the denotational semantics definition in Figure 6 because we require that all bound variables are distinct. 


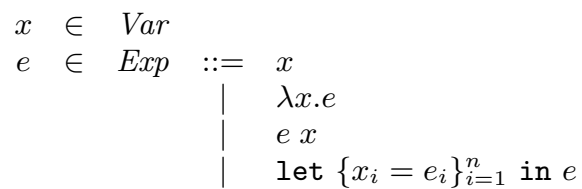

Figure 5: Syntax

\author{
$\llbracket x \rrbracket_{\rho}=\rho x$ \\ $\llbracket \lambda x . e \rrbracket_{\rho}=\operatorname{up}\left(\lambda \nu . \llbracket e \rrbracket_{\rho \sqcup\{x \mapsto \nu\}}\right)$ \\ $\llbracket e x \rrbracket_{\rho}=\mathrm{AP}_{D}^{\perp}\left(\llbracket e \rrbracket_{\rho}, \llbracket x \rrbracket_{\rho}\right)$ \\ $\llbracket$ let $\left\{x_{i}=e_{i}\right\}_{i=1}^{n}$ in $e \rrbracket_{\rho}=\llbracket e \rrbracket_{\left\{\left(x_{i} \mapsto e_{i}\right)_{i=1}^{n}\right\} \rho}$
}

\section{Figure 6: Denotational Semantics}

\subsection{A resourced denotational semantics}

A resourced denotational semantics is also defined in [7], where the meaning of an expression depends on the number of available resources. For this a new domain of values, and the corresponding environments, are needed.

A resourced environment is a function mapping variables into functions from resources to values,

$$
\sigma \in C E n v=\operatorname{Var} \rightarrow[C \rightarrow \text { CValue }],
$$

where $C$ Value is some appropiate resourced domain, i.e.,

$$
\text { CValue }=[[C \rightarrow \text { CValue }] \rightarrow[C \rightarrow \text { CValue }]]_{\perp} .
$$

Notice that CValue corresponds to the resourced domain $E$ constructed in Section 2 .

An ordering is defined on resourced environments too, such that if $\sigma$ is less or equal than $\sigma^{\prime}$ then, for any number of available resources, $\sigma^{\prime}$ may bind more variables than $\sigma$, but otherwise is equal to $\sigma$ : Let $\sigma, \sigma^{\prime} \in C E n v$ be resourced environments, $\sigma$ is less or equal than $\sigma^{\prime}$ (denoted as $\left.\sigma \leq \sigma^{\prime}\right)$ iff $\forall x \in \operatorname{Var}$ and $\forall m \in \mathbb{N}$

$$
\sigma x\left(S^{m}(\perp)\right) \neq \perp \Rightarrow \sigma x\left(S^{m}(\perp)\right)=\sigma^{\prime} x\left(S^{m}(\perp)\right) .
$$

The resourced semantics focuses on approximations to the semantics of Figure 6. The semantic function $\mathcal{N} \llbracket-\rrbracket$ : $E x p \rightarrow C E n v \rightarrow[C \rightarrow C$ Value $]$ is given in Figure 7 .

The function $\mathcal{N}\{\{-\}\}:$ Heap $\rightarrow$ CEnv $\rightarrow$ CEnv is defined analogously to $\{\{-\}\}$ :

$$
\mathcal{N}\left\{\left\{\left(x_{i} \mapsto E_{i}\right)_{i=1}^{n}\right\} \sigma=\mu \sigma^{\prime} . \sigma \sqcup\left(x_{i} \mapsto \mathcal{N} \llbracket E_{i} \rrbracket_{\sigma^{\prime}}\right)_{i=1}^{n} .\right.
$$

\subsection{Equivalence}

In Section 3 we have shown how to relate values in Value with values in CValue, but we need to extend the notion of similarity to environments:

DeFinition 3. (Similarity of environments). Let $\rho \in$ Env be an environment and $\sigma \in C E n v$ be a resourced environment. $\rho$ and $\sigma$ are similar (denoted by $\rho \triangleleft \triangleright \sigma$ ) when

$$
\forall x \in \operatorname{Var} . \rho x \triangleleft \triangleright \sigma x\left(S^{\infty}\right) \text {. }
$$

Now we can prove the equivalence between the standard denotational semantics and the resourced one. More precisely, we prove that they produce similar values.

THEOREM 10. Let $e \in$ Exp be an expression and $\rho \in$ Env, $\sigma \in C E n v$ be similar environments (i.e., $\rho \triangleleft \triangleright \sigma$ ), then

$$
\llbracket e \rrbracket_{\rho} \triangleleft \triangleright \mathcal{N} \llbracket e \rrbracket_{\sigma}\left(S^{\infty}\right) .
$$

Proof. By structural induction on $e$ :

$\mathbf{e} \equiv \mathbf{x}$

By definition, $\llbracket x \rrbracket_{\rho}=\rho x$ and $\mathcal{N} \llbracket x \rrbracket_{\sigma}\left(S^{\infty}\right)=\sigma x\left(S^{\infty}\right)$.

By hypothesis $\rho \triangleleft \triangleright \sigma$, therefore:

$\llbracket x \rrbracket_{\rho}=\rho x \triangleleft \triangleright \sigma x\left(S^{\infty}\right)=\mathcal{N} \llbracket x \rrbracket_{\sigma}\left(S^{\infty}\right)$. $\mathbf{e} \equiv \lambda \mathbf{x} \cdot \mathbf{e}^{\prime}$

By definition, $\llbracket \lambda x \cdot e^{\prime} \rrbracket_{\rho}=\operatorname{up}\left(\lambda \nu \cdot \llbracket e^{\prime} \rrbracket_{\rho \sqcup\{x \mapsto \nu\}}\right) \neq \perp_{\text {Value }}$ and $\mathcal{N} \llbracket \lambda x . e^{\prime} \rrbracket_{\sigma}\left(S^{\infty}\right)=\operatorname{up}\left(\lambda \tau . \mathcal{N} \llbracket e^{\prime} \rrbracket_{\sigma \sqcup\{x \mapsto \tau\}}\right) \neq \perp_{\text {CValue }}$.

We have to prove that $\operatorname{up}\left(\lambda \nu . \llbracket e^{\prime} \rrbracket_{\rho \sqcup\{x \mapsto \nu\}}\right) \triangleleft \triangleright \operatorname{up}\left(\lambda \tau . \mathcal{N} \llbracket e^{\prime} \rrbracket_{\sigma \sqcup\{x \mapsto \tau\}}\right)$.

We use the alternative characterization of similarity (Proposition 9). Let $v \in$ Value and $f \in C \rightarrow C$ Value such that $v \triangleleft \triangleright f\left(S^{\infty}\right)$, then:

$\operatorname{AP}_{D}^{\perp}\left(\operatorname{up}\left(\lambda \nu \cdot \llbracket e^{\prime} \rrbracket_{\rho \sqcup\{x \mapsto \nu\}}\right), v\right)=\llbracket e^{\prime} \rrbracket_{\rho \sqcup\{x \mapsto v\}}$ and

$\operatorname{AP}_{E}^{\perp}\left(\operatorname{up}\left(\lambda \tau . \mathcal{N} \llbracket e^{\prime} \rrbracket_{\sigma \sqcup\{x \mapsto \tau\}}\right), f, S^{\infty}\right)=\mathcal{N} \llbracket e^{\prime} \rrbracket_{\sigma \sqcup\{x \mapsto f\}}\left(S^{\infty}\right)$. If $\rho^{\prime}=\rho \sqcup\{x \mapsto v\} \quad \triangleleft \triangleright \sigma \sqcup\{x \mapsto f\}=\sigma^{\prime}$, then, by induction hypothesis, we get the desired result.

Let us prove that $\rho^{\prime} \triangleleft \triangleright \sigma^{\prime}$ :

- If $y \neq x$ then $\rho^{\prime} y=\rho y \triangleleft \triangleright \sigma y\left(S^{\infty}\right)=\sigma^{\prime} y$, because $\rho \triangleleft \triangleright \sigma$.

- If $y=x$ then $\rho^{\prime} y=v \triangleleft \triangleright f\left(S^{\infty}\right)=\sigma^{\prime} y$, by hypothesis.

Thus, $\llbracket e^{\prime} \rrbracket \rho^{\prime} \triangleleft \triangleright \mathcal{N} \llbracket e^{\prime} \rrbracket_{\sigma^{\prime}}\left(S^{\infty}\right)$.

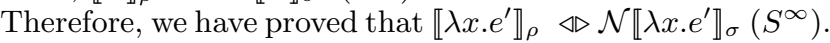

$\mathbf{e} \equiv \mathbf{e}^{\prime} \mathbf{x}$

By definition, $\llbracket e^{\prime} x \rrbracket_{\rho}=\operatorname{AP}_{D}^{\perp}\left(\llbracket e^{\prime} \rrbracket_{\rho}, \llbracket x \rrbracket_{\rho}\right)$ and

$\mathcal{N} \llbracket e^{\prime} x \rrbracket_{\sigma}\left(S^{\infty}\right)=\mathrm{AP}_{E}^{\perp}\left(\mathcal{N} \llbracket e^{\prime} \rrbracket_{\sigma} S^{\infty}, \mathcal{N} \llbracket x \rrbracket_{\sigma}, S^{\infty}\right)$.

Thus, we have to prove that

$\mathrm{AP}_{D}^{\perp}\left(\llbracket e^{\prime} \rrbracket_{\rho}, \llbracket x \rrbracket_{\rho}\right) \triangleleft \triangleright \mathrm{AP}_{E}^{\perp}\left(\mathcal{N} \llbracket e^{\prime} \rrbracket_{\sigma}\left(S^{\infty}\right), \mathcal{N} \llbracket x \rrbracket_{\sigma}, S^{\infty}\right)$

By induction hypothesis, $\llbracket e^{\prime} \rrbracket_{\rho} \triangleleft \triangleright \mathcal{N} \llbracket e^{\prime} \rrbracket_{\sigma}\left(S^{\infty}\right)$. Then by Corollary 8 there are two cases:

- Case 1: $\llbracket e^{\prime} \rrbracket_{\rho}=\perp_{\text {Value }}$ and $\mathcal{N} \llbracket e^{\prime} \rrbracket_{\sigma}\left(S^{\infty}\right)=\perp_{\text {CValue }}$. $\llbracket e^{\prime} x \rrbracket_{\rho}=\mathrm{AP}_{D}^{\perp}\left(\llbracket e^{\prime} \rrbracket \rho, \llbracket x \rrbracket_{\rho}\right)=\mathrm{AP}_{D}^{\perp}\left(\perp_{\text {Value }}, \llbracket x \rrbracket_{\rho}\right)=\perp_{\text {Value }}$ $\varangle \triangleright \perp_{\text {CValue }}=\mathrm{AP}_{E}^{\perp}\left(\perp_{\text {CValue }}, \mathcal{N} \llbracket x \rrbracket_{\sigma}, S^{\infty}\right)$ $=\operatorname{AP}_{E}^{\perp}\left(\mathcal{N} \llbracket e^{\prime} \rrbracket_{\sigma}\left(S^{\infty}\right), \mathcal{N} \llbracket x \rrbracket_{\sigma}, S^{\infty}\right)=\mathcal{N} \llbracket e^{\prime} x \rrbracket_{\sigma}\left(S^{\infty}\right)$.

- Case 2: $\perp_{\text {Value }} \neq \llbracket e^{\prime} \rrbracket_{\rho} \triangleleft \mathcal{N} \llbracket e^{\prime} \rrbracket_{\sigma}\left(S^{\infty}\right) \neq \perp_{C \text { Value }}$.

Thus, $\operatorname{AP}_{D}^{\perp}\left(\llbracket e^{\prime} \rrbracket_{\rho}, \llbracket x \rrbracket_{\rho}\right)=\llbracket e^{\prime} \rrbracket_{\rho}\left(\llbracket x \rrbracket_{\rho}\right)$ and

$\operatorname{AP}_{E}^{\perp}\left(\mathcal{N} \llbracket e^{\prime} \rrbracket_{\sigma}\left(S^{\infty}\right), \mathcal{N} \llbracket x \rrbracket_{\sigma}, S^{\infty}\right)=$ $\mathcal{N} \llbracket e^{\prime} \rrbracket_{\sigma}\left(S^{\infty}\right)\left(\mathcal{N} \llbracket x \rrbracket_{\sigma}\right)\left(S^{\infty}\right)$

By induction hypothesis $\llbracket x \rrbracket_{\rho} \triangleleft \triangleright \mathcal{N} \llbracket x \rrbracket_{\sigma}\left(S^{\infty}\right)$.

Therefore, by the alternative characterization for $\triangleleft \triangleright$ : $\llbracket e^{\prime} \rrbracket_{\rho}\left(\llbracket x \rrbracket_{\rho}\right) \triangleleft \triangleright \mathcal{N} \llbracket e^{\prime} \rrbracket_{\sigma}\left(S^{\infty}\right)\left(\mathcal{N} \llbracket x \rrbracket_{\sigma}\right)\left(S^{\infty}\right)$.

So that $\llbracket e^{\prime} x \rrbracket_{\rho} \triangleleft \triangleright \mathcal{N} \llbracket e^{\prime} x \rrbracket_{\sigma}\left(S^{\infty}\right)$.

$\mathbf{e} \equiv \operatorname{let}\left\{\mathbf{x}_{\mathbf{i}}=\mathbf{e}_{\mathbf{i}}\right\}_{\mathbf{i}=\mathbf{1}}^{\mathbf{n}}$ in $\mathbf{e}^{\prime}$

By definition:

【let $\left\{x_{i}=e_{i}\right\}_{i=1}^{n}$ in $e^{\prime} \rrbracket_{\rho}=\llbracket e^{\prime} \rrbracket_{\left\{\left(x_{i} \mapsto e_{i}\right)_{i=1}^{n}\right\} \rho \text { and }}$ $\mathcal{N} \llbracket$ let $\left\{x_{i}=e_{i}\right\}_{i=1}^{n}$ in $e^{\prime} \rrbracket_{\sigma}\left(S^{\infty}\right)=$

$$
\mathcal{N} \llbracket e^{\prime} \rrbracket_{\mathcal{N}\left\{\left(x_{i} \mapsto e_{i}\right)_{i=1}^{n}\right\} \sigma}\left(S^{\infty}\right) .
$$

Using fix point techniques it can be proved that if $\rho \triangleleft \triangleright \sigma$ then $\{\Gamma\}\} \rho \triangleleft \mathcal{N}\{\{\Gamma\}\}$ for any heap $\Gamma$ consistent with $\rho$ and $\sigma$. Thus, $\left\{\left(x_{i} \mapsto e_{i}\right)_{i=1}^{n}\right\} \rho \triangleleft \triangleright \mathcal{N}\left\{\left\{\left(x_{i} \mapsto e_{i}\right)_{i=1}^{n}\right\} \sigma \sigma\right.$.

By induction hypothesis:

$\llbracket e^{\prime} \rrbracket_{\left\{\left(x_{i} \mapsto e_{i}\right)_{i=1}^{n}\right\} \rho \rho} \triangleleft \mathcal{N} \llbracket e^{\prime} \rrbracket_{\mathcal{N}\left\{\left\{\left(x_{i} \mapsto e_{i}\right)_{i=1}^{n}\right\} \sigma\right.}\left(S^{\infty}\right)$.

Therefore, we obtain that

【let $\left\{x_{i}=e_{i}\right\}_{i=1}^{n}$ in $e^{\prime} \rrbracket_{\rho} \triangleleft \triangleright$

$$
\mathcal{N} \llbracket \text { let }\left\{x_{i}=e_{i}\right\}_{i=1}^{n} \text { in } e^{\prime} \rrbracket_{\sigma}\left(S^{\infty}\right) \text {. }
$$

This result allows to prove the computational adequacy of the natural semantics with respect to the denotational one. 


$$
\begin{aligned}
\mathcal{N} \llbracket e \rrbracket_{\sigma}(\perp) & =\perp \\
\mathcal{N} \llbracket x \rrbracket_{\sigma}\left(S^{k+1}(\perp)\right) & =\sigma x\left(S^{k}(\perp)\right) \\
\mathcal{N} \llbracket \lambda x . e \rrbracket_{\sigma}\left(S^{k+1}(\perp)\right) & =\operatorname{up}\left(\lambda \tau \cdot \mathcal{N} \llbracket e \rrbracket_{\sigma \sqcup\{x \mapsto \tau\}}\right) \text { where } \tau: C \rightarrow \text { CValue } \\
\mathcal{N} \llbracket e x \rrbracket_{\sigma}\left(S^{k+1}(\perp)\right) & =\operatorname{AP}_{E}^{\perp}\left(\mathcal{N} \llbracket e \rrbracket_{\sigma}\left(S^{k}(\perp)\right), \mathcal{N} \llbracket x \rrbracket_{\sigma}, S^{k}(\perp)\right) \\
\mathcal{N} \llbracket \text { let }\left\{x_{i}=e_{i}\right\}_{i=1}^{n} \text { in } e \rrbracket_{\sigma}\left(S^{k+1}(\perp)\right) & =\mathcal{N} \llbracket e \rrbracket_{\mathcal{N}\left\{\left\{\left(x_{i} \mapsto e_{i}\right)_{i=1}^{n}\right\} \sigma\right.}\left(S^{k}(\perp)\right)
\end{aligned}
$$

Figure 7: Resourced Denotational Semantics

\section{CONCLUSIONS AND FUTURE WORK}

We have tackled the problem of constructing a semantic domain for representing denotationally a resourced semantics. Abramsky and Ong defined in [2] an initial solution for the domain equation $D=[D \rightarrow D]_{\perp}$ suitable for defining a denotational semantics for a lazy $\lambda$-calculus. Following their schema, we have constructed the initial solution of the domain equation $E=[[C \rightarrow E] \rightarrow[C \rightarrow E]]_{\perp}$. We define a set of constructions on embeddings (adding a resource, lifting and function space), which can be cleanly defined by using function sections, and they simplify the presentation of the construction of $E$ with respect to that of $D$ in [2].

A resourced function space like $E$ can be used to define a resourced denotational semantics that models the consumption of syntactic levels and thus, be nearer to syntax-oriented operational semantics based on rules, such as Launchbury's natural semantics for lazy evaluation. In fact, this is the approach taken by Launchbury in [7] in order to prove the computational adequacy of his natural semantics. But the equivalence between the standard and the resourced denotational semantics turns out to be not so easy to establish, because the semantic domains are different. The same problem can be found in [15].

To prove the computational adequacy it is only required that both semantics converge for the same expressions. However, in order to prove this result by structural induction a stronger property is needed, namely that both semantics produce values that behave "similarly". The problem arises in the application rule, because it depends on the semantics of the functional abstraction. Therefore, we have defined a similarity relation between the values of $D=[D \rightarrow D]_{\perp}$ and those of $E=[[C \rightarrow E] \rightarrow[C \rightarrow E]]_{\perp}$. Since a direct definition of the relation between $D$ and $E$ is not possible, we have first defined the similarity gradually between the approximation domains $D_{n}$ and $E_{n}$. Afterwards, we prove that this layered definition satisfies an applicative bisimulationlike property.

We are interested in studying more properties of the similarity relation, especially in the context of category theory. We also want to compare it with the notions of bisimulation and bisimilarity - particularly with Abramsky's applicative bisimulation [1] - and with observational or contextual equivalences, like those in [10] or [12].

\section{ACKNOWLEDGMENTS}

We are thankful to John Launchbury for his valuable comments, and appreciate very much the help of David de Frutos Escrig. This work is partially supported by the grants: TIN2009-14599-C03-01, BES-2007-16823, S2009/TIC-1465.

\section{REFERENCES}

[1] S. Abramsky. Research Topics in Functional Programming, chapter The Lazy Lambda Calculus, pages 65-116. Addison-Wesley, 1990.

[2] S. Abramsky and C.-H. L. Ong. Full abstraction in the lazy lambda calculus. Information and Computation, 105(2):159-267, 1993.

[3] C. Baker-Finch, D. King, and P. W. Trinder. An operational semantics for parallel lazy evaluation. In ACM International Conference on Functional Programming (ICFP'00), pages 162-173, 2000.

[4] H. P. Barendregt. The Lambda Calculus: Its Syntax and Semantics, volume 103 of Studies in Logic and the Foundations of Mathematics. North-Holland, 1984.

[5] G. Boudol, P. Curien, and C. Lavatelli. A semantics for lambda calculi with resources. Mathematical Structures in Computer Science, 9(4):437-482, 1999.

[6] C. Gunter and D. S. Scott. Semantic domains. In Handbook of Theoretical Computer Science, Volume B: Formal Models and Semantics, pages 633-674. Elsevier Science, 1990.

[7] J. Launchbury. A natural semantics for lazy evaluation. In ACM Symposium on Principles of Programming Languages (POPL'93), pages 144-154. ACM Press, 1993.

[8] A. Melton, D. A. Schmidt, and G. E. Strecker. Galois connections and computer science applications. In Category Theory and Computer Programming, pages 299-312. LNCS 240, Springer, 1986.

[9] K. Nakata and M. Hasegawa. Small-step and big-step semantics for call-by-need. Journal of Functional Programming, 19(6):699-722, 2009.

[10] G. D. Plotkin. Call-by-name, call-by-value and the $\lambda$-calculus. Journal of Theoretical Computer Science, 1(2):125-159, 1975.

[11] L. Sánchez-Gil, M. Hidalgo-Herrero, and Y. Ortega-Mallén. Trends in Functional Programming, volume 10, chapter An Operational Semantics for Distributed Lazy Evaluation, pages 65-80. Intellect, 2010.

[12] M. Schmidt-Schauğ. Equivalence of call-by-name and call-by-need for lambda-calculi with letrec. Technical report, Institut für Informatik. J. W. Goethe Universität Frankfurt am Main, Germany, 2006.

[13] D. S. Scott. Continuous lattices. In Toposes, Algebraic Geometry and Logic, pages 97-136. LNCS 274, Springer, 1972.

[14] P. Sestoft. Deriving a lazy abstract machine. Journal of Functional Programming, 7(3):231-264, 1997.

[15] M. van Eekelen and M. de Mol. Reflections on Type Theory, $\lambda$-calculus, and the Mind. Essays dedicated to Henk Barendregt on the Occasion of his 60th Birthday, chapter Proving Lazy Folklore with Mixed Lazy/Strict Semantics, pages 87-101. Radboud University Nijmegen, The Netherlands, 2007. 